\title{
Upregulation of Beclin-1 expression in DU-145 cells following low-frequency ultrasound irradiation combined with microbubbles
}

\author{
YU WANG, YI-NI CHEN, WEI ZHANG, YU YANG, E. SHEN and BING HU \\ Department of Ultrasound in Medicine, Shanghai Jiao Tong University Affiliated Sixth People's Hospital, \\ Shanghai Institute of Ultrasound in Medicine, Shanghai 200233, P.R. China
}

Received August 9, 2014; Accepted April 29, 2015

DOI: $10.3892 / 01.2015 .3509$

\begin{abstract}
Castration-resistant prostate cancer (PCa) is difficult to treat. Autophagy, which is an evolutionarily conserved mechanism, plays an important role in cancer development. The balance between cell death and survival in different stages varies in cancer development. The role of autophagy in $\mathrm{PCa}$ development has not yet been fully elucidated. Ultrasound may be of value in the treatment of PCa. The aim of the present study was to investigate the association between autophagy and ultrasound combined with microbubbles. The MTT assay was used to evaluate cell viability. Autophagy was observed by transmission electron microscopy. Reverse transcription-polymerase chain reaction and western blot analysis were used to assess the expression of autophagy-related genes. The results revealed that cell viability was significantly reduced by ultrasound combined with microbubbles in DU145 PCa cells. The present study demonstrated that ultrasound combined with microbubbles induced autophagy and autophagy-related DU-145 cell death. Notably, these findings highlighted additional mechanisms that suggest the potential of ultrasound-modulated autophagy as a novel therapeutic strategy for PCa.
\end{abstract}

\section{Introduction}

Prostate cancer (PCa) is one of the leading causes of cancer-related mortality worldwide. Early-stage $\mathrm{PCa}$ is androgen-dependent and may be treated effectively with androgen ablation therapy, radiation and/or surgery. However, all the patients eventually progress to an androgen-independent state, referred to as castration-resistant $\mathrm{PCa}$, resulting in

Correspondence to: Professor Bing Hu, Department of Ultrasound in Medicine, Shanghai Jiao Tong University Affiliated Sixth People's Hospital, Shanghai Institute of Ultrasound in Medicine, 600 Yi Shan Road, Shanghai 200233, P.R. China

E-mail: binghu1314@hotmail.com

Key words: ultrasound combined with microbubbles, autophagy, prostate cancer metastasis or death (1). At this stage, there are no effective treatment options available for PCa (2). Therefore, there is an urgent requirement for the development of novel treatment methods for PCa (3).

Ultrasound has been proven to be a useful therapeutic and diagnostic method, which is safe, non-invasive and cost-effective (4). Ultrasound has been widely used in cancer therapy and has been shown to mediate apoptosis through thermal, cavitation, sonoporation and sonochemical effects; however, the underlying mechanisms remain unclear (5). Furthermore, a number of experiments indicated that the effects of ultrasound may be enhanced by microbubbles $(6,7)$.

Autophagy maintains homeostasis in normal cells and is triggered as a protective response to stressful factors, including nutrient deprivation, oxidative stress and infection. It was previously demonstrated that disorders in autophagy may lead to cancer; this may also be used as a type of anticancer therapy by promoting autophagic cell death in tumors (8). However, since the role of autophagy in cell survival and death remains unclear, further studies are required to determine its function (9). The aim of the present study was to determine whether ultrasound is able to modify autophagy in PCa cells.

\section{Materials and methods}

Cell lines. The human DU-145 PCa cell line was obtained from the Cell Bank of the Chinese Academy of Sciences (Shanghai, China) and used in all the experiments. The cells were incubated at $37^{\circ} \mathrm{C}$ in $5 \% \mathrm{CO}_{2}$ and Dulbecco's modified Eagle's medium supplemented with $10 \%$ fetal bovine serum (Gibco-BRL, Grand Island, NY, USA) was used as the culture medium, which was replaced every second day.

Ultrasound apparatus and microbubbles. The experiment was performed using FS-450 ultrasonic processing (Shanghai Institute of Ultrasound in Medicine, Shanghai, China). In all the procedures, the probe frequency was fixed at $21 \mathrm{kHz}$, the intensity was $4.6 \mathrm{~mW} / \mathrm{cm}^{2}$ and the duty cycle was $20 \%$ (i.e., $2 \mathrm{sec}$ 'on' and $8 \mathrm{sec}$ 'off' time), with a total exposure time of $5 \mathrm{~min}$. The SonoVue ${ }^{\mathrm{TM}}$ microbubble echo-contrast agent (Bracco $\mathrm{SpA}$, Milan, Italy) was reconstituted in $5 \mathrm{ml}$ phosphate-buffered saline, $200 \mu \mathrm{l}$ of which was used in the experimental 
group (10). The cells were divided into two groups, namely the control group (CON; no treatment) and the group administered ultrasound combined with microbubbles (US + MB). Each test was repeated three times.

Measurement of cell proliferation by the MTT assay. Following treatment, the cells in each group were grown to $80 \%$ confluence in 96-well plates for $24 \mathrm{~h}$ and their viability was assessed with the MTT assay (Wellscan MK3; Ani Labsystems Ltd. OY, Vantaa, Finland). According to the manufacturer's instructions, MTT reagent $(50 \mu \mathrm{l})$ was incubated with the cells for $4 \mathrm{~h}$ at $37^{\circ} \mathrm{C}$. Subsequently, the MTT reagent was removed and $150 \mu 1$ dimethylsulfoxide was added to each well and agitated for $15 \mathrm{~min}$. The optical density was measured at a wavelength of $492 \mathrm{~nm}$ using a microculture plate reader (BioTek, Winooski, VT, USA). The result was calculated as follows: Cell viability $(\%)=\left(\right.$ Absorbance $_{\mathrm{US}+\mathrm{MB}} /$ Absorbance $\left._{\mathrm{CON}}\right) \times 100$. Cell viability was calculated based on the average percentage.

Transmission electron microscopy. Ultra-thin specimens of DU-145 cells from each group were prepared according to the conventional method (11) and were observed and photographed using transmission electron microscopy (Hitachi S-4800; Hitachi High-Technologies Corporation, Tokyo, Japan) at a magnification of x24,500. Autophagosomes were identified by the characteristic structure of a double- or multi-lamellar smooth membrane completely surrounding compressed mitochondria, or as membrane-bound electron-dense material.

Reverse transcription-polymerase chain reaction (RT-PCR) analysis. After having grown to $\sim 85 \%$ confluence, the cells were harvested for RNA isolation using TRIzol ${ }^{\circledR}$ reagent (Invitrogen Life Technologies, Carlsbad, CA, USA), according to the manufacturer's instructions. Following purification, RNA was subjected to quantitative PCR analysis using an iQ5 $5^{\mathrm{TM}}$ multicolor detection system (Bio-Rad, Hercules, CA, USA); GAPDH expression levels were used as control. The results of the relative expression levels were calculated using the relative quantitative $2^{-\Delta \Delta}$ cycle threshold method. The primer sequences are presented in Table I.

Western blot analysis. After having grown to $80 \%$ confluence, the cells were harvested using lysis buffer (Beyotime Institute of Biotechnology, Shanghai, China) and protein samples were collected and quantified by running on SDS-PAGE gel (Beyotime Institute of Biotechnology) and transferring onto a nitrocellulose membrane (Sigma-Aldrich, St. Louis, MO, USA). Protein samples were probed with the indicated primary antibody (dilution, 1:500; catalog no., sc-11427; Santa Cruz Biotechnology, Inc., Santa Cruz, CA, USA) overnight at $4^{\circ} \mathrm{C}$ and then incubated with secondary antibody (anti-mouse $\mathrm{IgG}$; dilution, 1:5,000; Beyotime Institute of Biotechnology) for $1 \mathrm{~h}$ at $25^{\circ} \mathrm{C} ; \beta$-actin was used as control. Protein was visualized using an enhanced chemiluminescence system and band intensity was quantified using Image $\mathbf{J}$ software (National Institutes of Health, Bethesda, MD, USA).

Statistical analysis. Independent Student's t-tests were performed using SPSS 13.0 software (SPSS Inc., Chicago, IL, USA). $\mathrm{P}<0.05$ was considered to indicate statistically significant
Table I. Oligonucleotide sequences for reverse transcription polymerase chain reaction.

\begin{tabular}{lc}
\hline Gene & \multicolumn{1}{c}{ Primer sequence $\left(5^{\prime}-3^{\prime}\right)$} \\
\hline$B E C N-1$ & \\
Forward & ATGGAGGGGTCTAAGGCGTC \\
Reverse & TGGGCTGTGGTAAGTAATGGA \\
GAPDH & \\
Forward & \\
Reverse & AATGGATTTGGACGCATTGGT \\
\hline
\end{tabular}

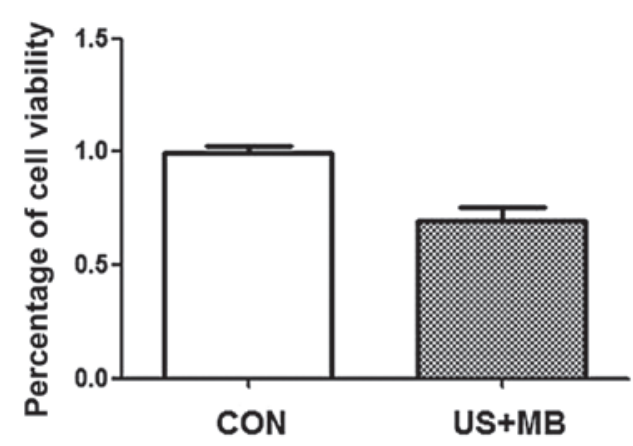

Figure 1. Cell proliferation in the CON and US + MB groups at $24 \mathrm{~h}$ after treatment. The percentage of cell viability in the two groups was calculated based on the OD measurement. CON, control; US + MB, ultrasound with microbubbles; OD, optical density.

differences between the CON and US + MB groups. All the experiments were performed at least 3 times. The results of the statistical analyses are presented as means \pm standard deviation.

\section{Results}

Measurement of cell proliferation. The MTT assay was used to evaluate cell viability. The percentage of cell viability was $99.2 \pm 7.5 \%$ in the CON and $69.3 \pm 14.7 \%$ in the US + MB group (Fig. 1). Therefore, DU-145 cell growth was inhibited by ultrasound combined with microbubbles.

Analysis of autophagosomes by transmission electron microscopy. Autophagosomes contain organelles or other decomposed residues and have a characteristic double- or multi-membrane structure. The number of autophagosomes under 10 different vision fields at a magnification of $\mathrm{x} 13,500$ was calculated in a blinded manner. Compared with $\mathrm{CON}$, the number of autophagosomes was significantly increased in the US + MB group (0.2 \pm 0.42 vs. $2.6 \pm 2.12$, respectively; $\mathrm{P}<0.05$ ) (Fig. 2 ).

Beclin-1 induces autophagy in DU-145 cells following ultrasound irradiation. Compared with the CON group, the mRNA level of the autophagy-related gene Beclin-1 was significantly increased in the DU-145 cells of the US + MB group (1.01 \pm 0.03 vs. $1.23 \pm 0.08$, respectively; $\mathrm{P}<0.05$; Fig. 3 ).

Western blot analysis of the Beclin-1 protein. The Beclin-1 protein expresion was assessed by western blotting and was 


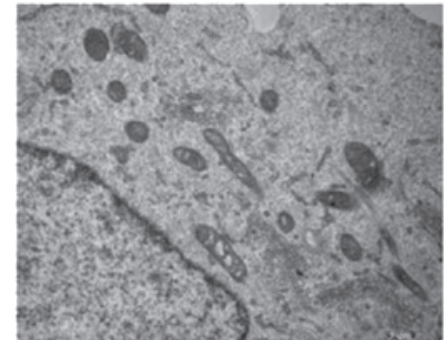

CON

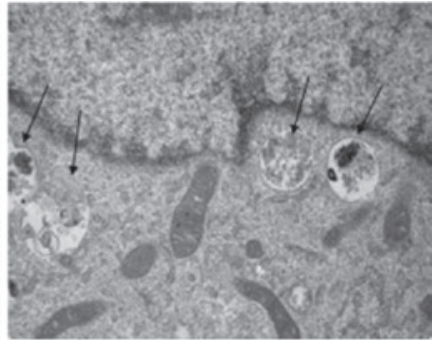

US+MB
Figure 2. Autophagy was significantly increased in the DU-145 cells of the $\mathrm{US}+\mathrm{MB}$ group compared with the CON group at $24 \mathrm{~h}$ after treatment (magnification, $\mathrm{x} 13,500)$. CON, control; US + MB, ultrasound with microbubbles.

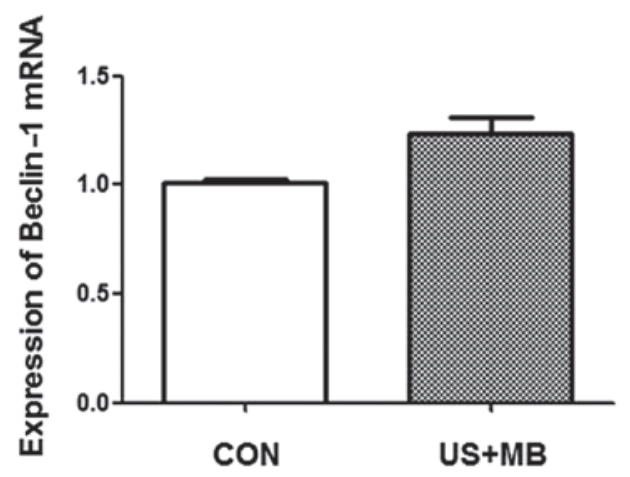

Figure 3. Expression of Beclin-1 mRNA in the CON and US + MB groups was determined using reverse transcription-polymerase chain reaction and was found to be increased in the DU-145 cells of the US + MB group following ultrasound irradiation. CON, control; US + MB, ultrasound with microbubbles.

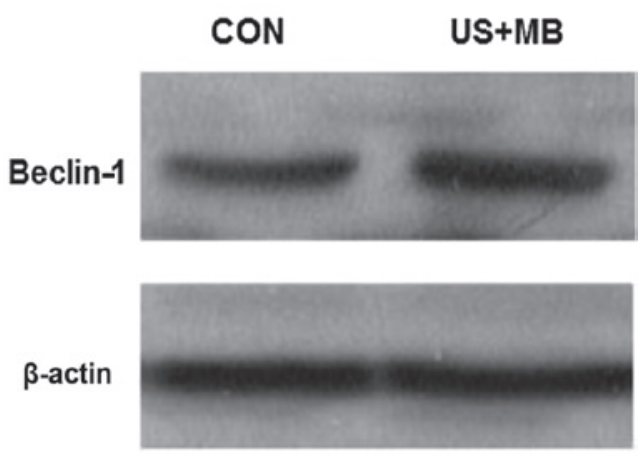

Figure 4. Expression of Beclin-1 protein in the CON and US + MB groups was determined using western blotting and was found to be increased in the DU-145 cells of the US + MB group following ultrasound irradiation. CON, control; US + MB, ultrasound with microbubbles.

found to be higher in the DU-145 cells of the US +MB group compared with the CON group (Fig. 4). Thus, ultrasound combined with microbubbles increased the expression level of the Beclin-1 protein.

\section{Discussion}

Autophagy, a highly conserved mechanism present in almost all species, constitutes an important part of the degradation/recirculation system, which is widely encountered from simple unicellular organisms and plants to mammalian cells (12-14). Autophagy is the main channel for the degradation of intact organelles and macromolecular protein and autophagy disorders are closely associated with the occurrence, development and outcome of cancer. Autophagosome formation is associated with autophagy-related genes (12-14). In the present study, it was demonstrated that there were more autophagosomes in the DU-145 cells of the US + MB group compared with the CON group $(\mathrm{P}<0.05)$. The autophagosomes of the DU-145 cells in the US + MB group exhibited typical characteristics, with double- or multi-membrane structures containing organelles or other decomposed residues (Fig. 2), indicating that ultrasound combined with microbubbles may induce autophagy in PCa cells.

Autophagy is regulated by a series of autophagy-related genes and changes in autophagy may occur in various human diseases. Liang et al (15) made the landmark discovery that cancer is genetically linked to impaired autophagy and that Beclin-1 is a phylogenetically conserved protein essential for autophagy. Beclin-1 monoallelic deletion on chromosome locus $17 \mathrm{q} 21$ occurs in $40-75 \%$ of human PCa, ovarian and breast cancer cases (16). Beclin-1 in mammals is homologous with autophagy-related gene 6 in yeast. Increased expression of Beclin-1 in mammalian cells may stimulate autophagy. Beclin-1-mediated autophagy maintains cellular homeostasis by adjusting the level of recycling and reuse of macromolecular materials. The Beclin-1 gene is considered to be a type of tumor suppressor gene. As demonstrated by RT-PCR and western blot analysis (Figs. 3 and 4) in the present study, DU-145 cells treated with ultrasound with microbubbles exhibited an increase in Beclin-1 expression, which favored autophagy (Fig. 2)

An increasing number of studies on the biological effect of ultrasound have indicated that the effects of ultrasound reach far beyond its classic role in diagnosis (17-22). Ultrasound therapy may be divided into different types according to different frequencies. The mechanisms of conventional low-frequency ultrasound in the treatment of tumors include the cytotoxic, apoptosis-promoting and sensitization effects of chemotherapy. At present, the majority of studies investigate the effect of low-frequency ultrasound on the biological behavior of tumor cells at the genetic level. Tabuchi et al (23) observed that 193 genes were downregulated and 201 were upregulated in tumor cells following irradiation by low-frequency ultrasound. These genes are associated with cell growth, proliferation, apoptosis, movement, polymorphism and death (24).

Low-frequency ultrasound has certain advantages in disease treatment. However, studies (25-28) on low-frequency ultrasound therapy are at an early stage and certain areas require further investigation: i) The mechanisms underlying the role of ultrasound in the treatment of certain diseases remain unclear; ii) the optimal parameters are under debate; iii) it has not been definitively established how to combine ultrasound with other methods of treatment; iv) the development of low-frequency ultrasound instruments cannot yet meet the personalized requirements of disease treatment; v) ultrasound cannot be concomitantly used for diagnosis and treatment; vi) the majority of the studies are currently at the laboratory stage and ultrasound has not yet been widely applied 
in clinical practice. Further studies may lead to low-frequency ultrasound becoming a highly efficient, convenient and widely used treatment method in the clinical setting.

In conclusion, the present study demonstrated that ultrasound combined with microbubbles induced autophagy through the upregulation of Beclin-1 in DU-145 PCa cells. Additionally, ultrasound combined with microbubbles significantly suppressed DU-145 cell growth. Based on these results, ultrasound combined with microbubbles shows considerable promise as a treatment for androgen-independent $\mathrm{PCa}$. However, the underlying mechanisms require further investigation.

\section{Acknowledgements}

This study was supported by grants from the National Natural Science Foundation of China (nos. 81271597 and 81401421) and the Major Infrastructure Projects of Shanghai Science and Technology (no. 10JC1412600).

\section{References}

1. Chaturvedi S and Garcia JA: Novel agents in the management of castration resistant prostate cancer. J Carcinog. 13: 5, 2014.

2. Shin SW, Kim SY and Park JW: Autophagy inhibition enhances ursolic acid-induced apoptosis in PC 3 cells. Biochim Biophys Acta 1823: 451-457, 2012.

3. Jácome-Pita F, Sánchez-Salas R, Barret E, Amaruch N, Gonzalez-Enguita $\mathrm{C}$ and Cathelineau X: Focal therapy in prostate cancer: the current situation. Ecancermedicalscience 8: 435, 2014.

4. Franco de Oliveira R, Pires Oliveira DA and Soares CP: Effect of low-intensity pulsed ultrasound on 1929 fibroblasts. Arch Med Sci 7: 224-229, 2011.

5. Feng Y,Tian Z and Wan M: Bioeffects of low-intensity ultrasound in vitro: apoptosis, protein profile alteration and potential molecular mechanism. J Ultrasound Med 29: 963-974, 2010.

6. Wang Y, Bai WK, Shen E and Hu B: Sonoporation by low-frequency and low-power ultrasound enhances chemotherapeutic efficacy in prostate cancer cells in vitro. Oncol Lett 6 : 495-498, 2013

7. Yang SL, Tang KQ, Bai WK, Shen E, Zhao YW, Lin YD, Nan SL and Bing $\mathrm{H}$ : Effects of low-frequency ultrasound combined with microbubbles on benign prostate hyperplasia. Can Urol Assoc J 7: E681-E686, 2013.

8. Law BY, Chan WK, Xu SW, Wang JR, Bai LP, Liu L and Wong VK: Natural small-molecule enhancers of autophagy induce autophagic cell death in apoptosis-defective cells. Sci Rep 4: 5510, 2014

9. Wei MF, Chen MW, Chen KC, Lou PJ, Lin SY, Hung SC, Hsiao M, Yao CJ and Shieh MJ: Autophagy promotes resistance to photodynamic therapy-induced apoptosis selectively in colorectal cancer stem-like cells. Autophagy 10: 1179-1192, 2014.
10. Bai WK, Yang SL, Shen E, et al: Treatment of PC-3 cells with ultrasound combined with microbubbles induces distinct alterations in the expression of Bcl-2 and Bax. Chin Sci Bull 58: 3535-3540, 2013.

11. Fan X, Wang J, Hou J, et al: Berberine alleviates ox-LDL induced inflammatory factors by up-regulation of autophagy via AMPK/mTOR signaling pathway. J Transl Med 13: 92, 2015.

12. Yorimitsu T and Klionsky DJ: Autophagy: Molecular machinery for self-eating. Cell Death Differ 12 (Suppl 2): 1542-1552, 2005.

13. Xie Z, Nair U and Klionsky DJ: Atg8 controls phagophore expansion during autophagosome formation. Mol Biol Cell 19: 3290-3298, 2008 .

14. Shintani T and Klionsky DJ: Autophagy in health and disease: Adouble-edged sword. Science 306: 990-995, 2004.

15. Liang XH, Jackson S, Seaman M, Brown K, Kempkes B, Hibshoosh $\mathrm{H}$ and Levine B: Induction of autophagy and inhibition of tumorigenesis by Beclin-1. Nature 402: 672-676, 1999.

16. Aita VM, Liang XH, Murty VV, Pincus DL, Yu W, Cayanis E, Kalachikov S, Gilliam TC and Levine B: Cloning and genomic organization of Beclin-1, a candidate tumor suppressor gene on chromosome 17q21. Genomics 59: 59-65, 1999.

17. Tsivgoulis G, Eggers J, Ribo M, et al: Safety and efficacy of ultrasound-enhanced thrombolysis: a comprehensive review and meta-analysis of randomized and nonrandomized studies. Stroke 41: 280-287, 2010.

18. Hensel K, Mienkina MP and Schmitz G: Analysis of ultrasound fields in cell culture wells for in vitro ultrasound therapy experiments. Ultrasound Med Biol 37: 2105-2115, 2011.

19. Rapoport NY, Nam KH, Gao Z and Kennedy A: Application of ultrasound for targeted nanotherapy of malignant tumors. Acoust Phys 55: 594-601, 2009.

20. Kawai N and Iino M: Molecular damage to membrane proteins induced by ultrasound. Ultrasound Med Biol 29: 609-614, 2003.

21. Marentis TC, Kusler B, Yaralioglu GG, et al: Microfluidic sonicator for real-time disruption of eukaryotic cells and bacterial spores for DNA analysis. Ultrasound Med Biol 31: 1265-1277, 2005.

22. Nyborg WL, Leighton TG, Miller D, et al: Nonthermal issues: cavitation - its nature, detection and measurement. Ultrasound Med Biol 6: 11-16, 1998

23. Tabuchi Y, Takasaki I, Zhao QL, et al: Genetic networks responsive to low-intensity pulsed ultrasound in human lymphoma U937 cells. Cancer Lett 270: 286-294, 2008.

24. Xu WP, Shen E, Bai WK, Wang Y and Hu B: Enhanced antitumor effects of low-frequency ultrasound and microbubbles in combination with simvastatin by downregulating caveolin-1 in prostatic DU145 cells. Oncol Lett 7: 2142-2148, 2014.

25. Maruani A, Boucaud A, Perrodeau E, et al: Low-frequency ultrasound sonophoresis to increase the efficiency of topical steroids: a pilot randomized study of humans. Int J Pharm 395: 84-90, 2010.

26. Liu HL, Chen WS, Chen JS, et al: Cavitation-enhanced ultrasound thermal therapy by combined low- and high-frequency ultrasound exposure. Ultrasound Med Biol 32: 759-767, 2006

27. Ren ST, Liao YR, Kang XN, et al: The antitumor effect of a new docetaxel-loaded microbubble combined with low-frequency ultrasound in vitro: Preparation and parameter analysis. Pharm Res 30: 1574-1585, 2013.

28. Yang SL, Tang KQ, Bai WK, et al: Combined low-frequency ultrasound and microbubble contrast agent for the treatment of benign prostatic hyperplasia. J Endourol 27: 1020-1026, 2013. 